\title{
On meet-combination of logics
}

\author{
A. Sernadas C. Sernadas J. Rasga \\ Dep. Matemática, Instituto Superior Técnico, Universidade Técnica de Lisboa \\ SQIG, Instituto de Telecomunicações, Portugal \\ $\{$ acs,css,jfr $\} @$ math.ist.utl.pt
}

May 18, 2011, revised September 9, 2011

\begin{abstract}
When combining logics while imposing the sharing of connectives, the result is frequently inconsistent. In fact, in fibring, fusion and other forms of combination reported in the literature, each shared connective inherits the logical properties of each of its components. A new form of combining logics (meet-combination) is proposed where such a connective inherits only the common logical properties of its components. The conservative nature of the proposed combination is shown to hold without provisos. Preservation of soundness and completeness is also proved. Illustrations are provided involving classical, intuitionistic and modal logics.
\end{abstract}

Keywords: combined logics, combined connectives.

\section{Introduction}

When combining logics one frequently wants to impose some interaction between connectives ${ }^{1}$. For instance, in the logic $\mathcal{L}$ resulting from the fibring [5] of any two given logics $\mathcal{L}_{1}$ and $\mathcal{L}_{2}$ (where one finds all the inference rules from those two logics), if one wants the sharing of two constructors $c_{1}$ and $c_{2}$ with the same arity, the shared constructor $\left\langle c_{1} c_{2}\right\rangle$ in $\mathcal{L}$ enjoys the logical properties inherited from $c_{1}$ together with those inherited from $c_{2}$. More concretely, if one shares a classical negation $\neg_{1}$ and an intuitionistic negation $\neg_{2}$, the resulting shared negation $\left\langle\neg_{1} \neg_{2}\right\rangle$ is classical. As expected, such a sharing may easily lead to inconsistency. For example, if one shares conjunction $\wedge_{1}$ and disjunction $\vee_{2}$, since the resulting shared connective $\left\langle\wedge_{1} \vee_{2}\right\rangle$ inherits the logical properties of conjunction and those of disjunction, we can infer $\varphi$ from $\psi$ for any formulas $\varphi, \psi$ in $\mathcal{L}:$

$$
\begin{array}{lll}
1 & \psi & \text { Hypothesis; } \\
2 & \psi\left\langle\wedge_{1} \vee_{2}\right\rangle \varphi & \text { Disjunction introduction; } \\
3 & \varphi & \text { Conjunction elimination. }
\end{array}
$$

One may wonder if it would not be better instead to endow the combined constructor only with the common logical properties of the component constructors. For instance, since both conjunction and disjunction are associative

\footnotetext{
${ }^{1}$ In a general sense, including, besides the propositional connectives, also modal operators and other language constructors.
} 
their combination should also be associative. On the other hand, since strong elimination holds for conjunction but not for disjunction, it should not hold for their combination.

As we conjectured in [7], we expect in this way to be led to a different way of combining logics, at least with respect to the behavior of the combined constructors, hopefully avoiding inconsistency in more situations.

Following this idea, we explain herein how to meet-combine logics and establish key transference results (preservation of completeness and soundness). We also show that, as envisaged, meet-combination is always a conservative extension of the combined logics and, so, that it preserves consistency. The proposed construction is illustrated with the combination of classical logic and intuitionistic logic, and with the combination of two modal logics.

For the sake of simplicity, we assume that the logics to be combined have a propositional nature ${ }^{2}$, are endowed with a Hilbert calculus and matrix semantics, and contain verum and falsum. Such suitable logics are described in Section 2 .

The means for meet-combining (language, calculus and semantics) any two given suitable logics are presented in Section 3. Every constructor of the resulting language is a pair of constructors of the same arity, one from each of the two original logics being combined. Moreover, each constructor of each original logic is embedded in the resulting signature by pairing (meet-combining) it with the verum from the other original logic. In the resulting calculus one finds the rules ported from the two given logics (via the embeddings mentioned above), rules imposing that the meet-combined constructors inherit the common properties of their components and only those common properties, and rules imposing the propagation of falsum. Every matrix of the resulting logic is just the product of a matrix from one of the original logics with a matrix of the other. In this way, the resulting logic is an enrichment of each of the original logics (via the relevant embedding).

In Section 4 we show the preservation of soundness and completeness, as well as the conservative nature of the enrichments and, as an immediate corollary, the preservation of consistency.

Examples of meet-combination are presented, analyzed and compared with fibring in Section 5. Finally, in Section 6 we assess what was achieved and speculate on future work.

\section{Suitable logics}

For the purposes of this paper, by a logic we mean a triple $\mathcal{L}=(\Sigma, \Delta, \mathcal{M})$ where:

- The signature $\Sigma$ is a family $\left\{\Sigma_{n}\right\}_{n \in \mathbb{N}}$ with each $\Sigma_{n}$ being a finite set of $n$-ary language constructors. Formulas are built as usual with these constructors and the schema variables in $\Xi=\left\{\xi_{k} \mid k \in \mathbb{N}\right\}$. We use $L$ and $L(\Xi)$ for denoting the set of concrete formulas ${ }^{3}$ and the set of all

\footnotetext{
${ }^{2}$ That is, with no binding operators.

${ }^{3}$ Formulas without schema variables.
} 
formulas, respectively. If a formula contains schema variables we may emphasize the fact by saying that it is a schema formula.

- The Hilbert calculus $\Delta$ is a set of finitary rules of the form

$$
\frac{\alpha_{1} \quad \ldots \quad \alpha_{m}}{\beta}
$$

where formulas $\alpha_{1}, \ldots, \alpha_{m}$ are said to be the premises of the rule and formula $\beta$ is said to be its conclusion. A rule without premises is said to be axiomatic and its conclusion is said to be an axiom. Derivations are defined as usual for Hilbert calculi. We write

$$
\Gamma \vdash \varphi
$$

for stating that there is a derivation of formula $\varphi$ from set $\Gamma$ of hypotheses. When $\emptyset \vdash \varphi$ we say that $\varphi$ is a theorem and write simply $\vdash \varphi$.

- The matrix semantics $\mathcal{M}$ is a non-empty class of matrices over $\Sigma$. Recall that a matrix over $\Sigma$ is a pair $M=(\mathfrak{A}, D)$ where

$$
\mathfrak{A}=\left(A,\left\{\underline{c}: A^{n} \rightarrow A \mid c \in \Sigma_{n}\right\}_{n \in \mathbb{N}}\right)
$$

is an algebra over $\Sigma$ and $D \subseteq A$. The elements of $A$ are known as truth values and those of $D$ are the distinguished or designated ones. Denotation, satisfaction and entailment are as expected for matrix semantics. We write

$$
\llbracket \varphi \rrbracket_{\mathfrak{A} \rho}
$$

for the denotation of formula $\varphi$ by algebra $\mathfrak{A}$ for assignment $\rho: \Xi \rightarrow A$. Furthermore, when $\varphi$ is concrete we may write $\llbracket \varphi \rrbracket_{\mathfrak{A}}$ for $\llbracket \varphi \rrbracket_{\mathfrak{A} \rho}$ since the denotation is independent of the assignment. Matrix $M$ and assignment $\rho$ satisfy formula $\varphi$, written

$$
M, \rho \Vdash \varphi,
$$

if $\llbracket \varphi \rrbracket_{\mathfrak{A} \rho} \in D$. Set $\Gamma$ of formulas entails formula $\varphi$, written

$$
\Gamma \vDash \varphi,
$$

if $M, \rho \Vdash \varphi$ whenever $M, \rho \Vdash \Gamma$. When $\emptyset \vDash \varphi$ we say that $\varphi$ is valid and write simply $\vDash \varphi$.

The following result which relates satisfaction with substitution is needed in order to establish that entailment is closed for substitution.

Proposition 2.1 Let $\varphi \in L(\Xi), \sigma: \Xi \rightarrow L(\Xi)$ be a substitution, $M \in \mathcal{M}$ and $\rho$ an assignment over $M$. Then

$$
\llbracket \varphi \rrbracket_{\mathfrak{A} \rho_{\sigma}}=\llbracket \sigma(\varphi) \rrbracket_{\mathfrak{A} \rho}
$$

and

$$
M, \rho_{\sigma} \Vdash \varphi \text { if and only if } M, \rho \Vdash \sigma(\varphi),
$$

where $\rho_{\sigma}(\xi)=\llbracket \sigma(\xi) \rrbracket_{\mathfrak{A} \rho}$ for each $\xi \in \Xi$. 
We omit the proof since the first statement follows straightforwardly by induction and the latter is a direct consequence of the former.

\section{Proposition 2.2 (Closure for substitution)}

Let $\Gamma \cup\{\varphi\} \subset L(\Xi)$ and $\sigma: \Xi \rightarrow L(\Xi)$. Then

$$
\sigma(\Gamma) \vDash \sigma(\varphi) \text { whenever } \Gamma \vDash \varphi .
$$

Proof: Assume that $\Gamma \vDash \varphi$ and $M, \rho \Vdash \sigma(\gamma)$ for each $\gamma \in \Gamma$ where $M \in$ $\mathcal{M}$ and $\rho$ is an assignment over $M$. Then $M, \rho_{\sigma} \Vdash \gamma$ for each $\gamma \in \Gamma$ by Proposition 2.1. Hence, by the hypothesis, $M, \rho_{\sigma} \Vdash \varphi$ and, so, $M, \rho \Vdash \sigma(\varphi)$ by the same proposition.

QED

We need to work with logics fulfilling some additional assumptions. By a suitable logic we mean a logic such that:

(i) there is a concrete formula which is both a theorem and valid.

(ii) there is a concrete formula which is unsatisfiable (that is, no matrix satisfies it) and from which every formula is derivable.

Assumption (i) is fulfilled by every sound logic with at least an axiom. Assumption (ii) is a bit more restrictive. For instance, leaves out positive implicational logic. From now on, in each suitable logic, we assume chosen once and for all a formula fulfilling (i) that we call verum and denote by tt. Furthermore, we also assume chosen once and for all a formula fulfilling (ii) that we call falsum and denote by ff.

In the context of a suitable logic, for each $n \geq 1$, we introduce by abbreviation the $n$-ary connective $\mathbb{t}^{(n)}$ as follows:

$$
\mathrm{t}^{(n)}\left(\varphi_{1}, \ldots, \varphi_{n}\right)=\mathrm{t} .
$$

Moreover, we may write $\mathrm{t}^{(0)}$ for $\mathrm{t}$.

Given a suitable logic $\mathcal{L}=(\Sigma, \Delta, \mathcal{M})$, we assume without loss of generality that $\Sigma$ contains the constructors $\mathbb{t}$, ff and $\mathrm{t}^{(n)}$ for each $n \in \mathbb{N}^{+}$, as introduced above.

\section{Meet combination of suitable logics}

Given two suitable logics $\mathcal{L}_{1}=\left(\Sigma_{1}, \Delta_{1}, \mathcal{M}_{1}\right)$ and $\mathcal{L}_{2}=\left(\Sigma_{2}, \Delta_{2}, \mathcal{M}_{2}\right)$, the objective now is to define a logic

$$
\left\lceil\mathcal{L}_{1} \mathcal{L}_{2}\right\rceil=\left(\Sigma_{\lceil 12\rceil}, \Delta_{\lceil 12\rceil}, \mathcal{M}_{\lceil 12\rceil}\right)
$$

where one can also reason with and about the constructors inherited from $\mathcal{L}_{1}$ and $\mathcal{L}_{2}$ as well as their combinations. 


\section{Language}

The signature $\Sigma_{\lceil 12\rceil}$ is composed of all possible pairs of constructors in $\Sigma_{1}$ and $\Sigma_{2}$. More concretely,

$$
\Sigma_{\lceil 12\rceil}=\left\{\Sigma_{\lceil 12\rceil n}\right\}_{n \in \mathbb{N}}
$$

with

$$
\Sigma_{\lceil 12\rceil n}=\left\{\left\lceil c_{1} c_{2}\right\rceil \mid c_{1} \in \Sigma_{1 n}, c_{2} \in \Sigma_{2 n}\right\} .
$$

For any constructors $c_{1} \in \Sigma_{1}$ and $c_{2} \in \Sigma_{2}$ of the same arity, $\left\lceil c_{1} c_{2}\right\rceil$ is said to be their meet-combination. Moreover, $c_{1}$ and $c_{2}$ are said to be the first component and the second component of $\left\lceil c_{1} c_{2}\right\rceil$, respectively. Since this paper addresses only such meet-combined constructors, from now on we may refer to them simply as combined constructors. As expected, we use $L_{\lceil 12\rceil}$ and $L_{\lceil 12\rceil}(\Xi)$ for denoting the set of concrete formulas and the set of all formulas over $\Sigma_{\lceil 12\rceil}$, respectively.

We look at signature $\Sigma_{\lceil 12\rceil}$ as an enrichment of $\Sigma_{1}$ via the embedding

$$
\eta_{1}: c_{1} \mapsto\left\lceil c_{1} \mathrm{t}_{2}^{(n)}\right\rceil \quad \text { for each } c_{1} \in \Sigma_{1 n} .
$$

Similarly, for $\Sigma_{2}$ we use the embedding

$$
\eta_{2}: c_{2} \mapsto\left\lceil\mathrm{t}_{1}^{(n)} c_{2}\right\rceil \text { for each } c_{2} \in \Sigma_{2 n} .
$$

Suitability assumption (i) is needed in order to ensure that these embeddings are logically faithful, as we shall prove in due course (at the end of Section 4). For the sake of lightness of notation, in the context of $\Sigma_{\lceil 12\rceil}$, from now on, we write

$$
c_{1} \text { for }\left\lceil c_{1} \mathrm{t}_{2}^{(n)}\right\rceil \text { when } c_{1} \in \Sigma_{1 n}
$$

and

$$
c_{2} \text { for }\left\lceil\mathrm{t}_{1}^{(n)} c_{2}\right\rceil \text { when } c_{2} \in \Sigma_{2 n} .
$$

We refer to these constructors as the inherited constructors and refer to the other constructors in $\Sigma_{\lceil 12\rceil}$ as the proper combined constructors.

In this vein, for $k=1,2$, we look at $L_{k}$ as a subset of $L_{\lceil 12\rceil}$ and at $L_{k}(\Xi)$ as a subset of $L_{\lceil 12\rceil}(\Xi)$.

Given a formula $\varphi$ over $\Sigma_{\lceil 12\rceil}$ and $k \in\{1,2\}$, we denote by

$$
\left.\varphi\right|_{k}
$$

the formula obtained from $\varphi$ by replacing every occurrence of each combined constructor (proper and inherited) by its $k$-th component.

\section{Calculus}

The calculus $\Delta_{[12\rceil}$ should be composed of the rules inherited from $\Delta_{1}$ (via the implicit embedding $\eta_{1}$ ) and the rules inherited from $\Delta_{2}$ (via the implicit embedding $\eta_{2}$ ), plus the rules imposing that each combined connective enjoys the common properties of its components and the rules for propagating falsum. 
At first sight one might be tempted to include in $\Delta_{\lceil 12\rceil}$ every rule in $\Delta_{1} \cup \Delta_{2}$. For instance, if modus ponens (MP) is a rule in $\Delta_{1}$ one would expect to find in $\Delta_{\lceil 12\rceil}$ the rule

$$
\frac{\xi_{1} \quad\left(\xi_{1} \supset_{1} \xi_{2}\right)}{\xi_{2}} .
$$

However, as we shall see in Section 5, this rule would not be sound for the semantics of meet-combination that we have in mind. Instead, we tag such a liberal rule (with a schema variable as conclusion), including in $\Delta_{\lceil 12\rceil}$ the following rule $\left(\mathrm{MP}_{c}\right)$

$$
\frac{\xi_{1} \quad\left(\xi_{1} \supset_{1} c\left(\xi_{3}, \ldots, \xi_{2+n}\right)\right)}{c\left(\xi_{3}, \ldots, \xi_{2+n}\right)} \text { for each } n \in \mathbb{N} \text { and } c \in \Sigma_{1 n} .
$$

Accordingly, $\Delta_{\lceil 12\rceil}$ contains the following rules:

- for $k=1,2$, the inherited rules from $\Delta_{k}$ :

- every non-liberal rule in $\Delta_{k}$;

- every tagging of every liberal rule $r$ of the form

$$
\begin{array}{ccc}
\alpha_{1} \quad \ldots \quad \alpha_{m} \\
\xi
\end{array}
$$

in $\Delta_{k}$, that is, the rule $r_{c}$ of the form

$$
\frac{\left.\left.\alpha_{1}\right|_{\beta_{c}} ^{\xi} \quad \cdots \quad \alpha_{m}\right|_{\beta_{c}} ^{\xi}}{\beta_{c}} \text { for each } n \in \mathbb{N} \text { and } c \in \Sigma_{k n}
$$

where $\beta_{c}=c\left(\xi_{j+1}, \ldots, \xi_{j+n}\right)$ with $j$ being the maximum of the indexes of the schema variables occurring in $r$;

- the lifting rule (in short LFT)

$$
\frac{\left.\left.\varphi\right|_{1} \varphi\right|_{2}}{\varphi}
$$

for each formula $\varphi \in L_{\lceil 12\rceil}(\Xi)$;

- the co-lifting rule (in short cLFT)

$$
\frac{\varphi}{\left.\varphi\right|_{k}}
$$

for each formula $\varphi \in L_{\lceil 12\rceil}(\Xi)$ and $k=1,2$;

- the falsum propagation rules (in short $\mathrm{FX}$ ) of the form

$$
\frac{\mathrm{ff}_{1}}{\mathrm{ff}_{2}} \text { and } \frac{\mathrm{ff}_{2}}{\mathrm{ff}_{1}} \text {. }
$$


The lifting rule is motivated by the idea that $\left\lceil c_{1} c_{2}\right\rceil$ inherits the common properties of $c_{1}$ and $c_{2}$. For instance,

$$
\frac{\left(\xi_{1} \wedge_{1} \xi_{2}\right) \equiv_{1}\left(\xi_{2} \wedge_{1} \xi_{1}\right) \quad\left(\xi_{1} \vee_{2} \xi_{2}\right) \equiv_{2}\left(\xi_{2} \vee_{2} \xi_{1}\right)}{\left(\xi_{1}\left\lceil\wedge_{1} \vee_{2}\right\rceil \xi_{2}\right)\left\lceil\equiv_{1} \equiv_{2}\right\rceil\left(\xi_{2}\left\lceil\wedge_{1} \vee_{2}\right\rceil \xi_{1}\right)}
$$

lifts commutativity of conjunction and disjunction to their combination.

The co-lifting rule is motivated by the idea that $\left\lceil c_{1} c_{2}\right\rceil$ should enjoy only the common properties of $c_{1}$ and $c_{2}$. In fact, this rule guarantees more. It guarantees that $\left\lceil c_{1} c_{2}\right\rceil$ enjoys only the common original properties of $c_{1}$ and $c_{2}$ because, in due course, we show that $L_{\lceil 12\rceil}$ is a conservative extension of $\mathcal{L}_{1}$ and $\mathcal{L}_{2}$.

Observe that although we may write, for example, $\supset_{1}$ for $\left\lceil\supset_{1} \mathrm{t}_{2}^{(2)}\right\rceil$, the lifting and co-lifting rules also apply to such inherited constructors. For example, we do have in the calculus of the meet-combination

$$
\frac{\xi_{1} \supset_{1} \xi_{2} \quad \xi_{1} \mathrm{t}_{2}^{(2)} \xi_{2}}{\xi_{1} \supset_{1} \xi_{2}}
$$

as an instance of LFT, as well as

$$
\frac{\xi_{1} \supset_{1} \xi_{2}}{\xi_{1} \supset_{1} \xi_{2}} \quad \frac{\xi_{1} \supset_{1} \xi_{2}}{\xi_{1} \mathrm{t}_{2}^{(2)} \xi_{2}}
$$

as instances of cLFT. Clearly, in these examples the application of LFT and cLFT to inherited formulas adds nothing to the calculus. However, in general, in a formula also with proper combined constructors, these rules produce effects and, so, when applying LFT and cLFT, the inherited constructors must be treated as combined constructors. For instance,

$$
\frac{\neg_{1}\left(\xi_{1} \supset_{1} \xi_{2}\right) \quad \square_{2}\left(\xi_{1} \mathrm{t}_{2}^{(2)} \xi_{2}\right)}{\left\lceil\neg_{1} \square_{2}\right\rceil\left(\xi_{1} \supset_{1} \xi_{2}\right)}
$$

is a non-vacuous instance of LFT.

\section{Semantics}

The semantics $\mathcal{M}_{\lceil 12\rceil}$ is the class of matrices over $\Sigma_{\lceil 12\rceil}$

$$
\left\{M_{1} \times M_{2} \mid M_{1} \in \mathcal{M}_{1} \text { and } M_{2} \in \mathcal{M}_{2}\right\}
$$

such that each

$$
M_{1} \times M_{2}=\left(\mathfrak{A}_{1} \times \mathfrak{A}_{2}, D_{1} \times D_{2}\right)
$$

where

$$
\mathfrak{A}_{1} \times \mathfrak{A}_{2}=\left(A_{1} \times A_{2},\left\{\underline{\left\lceil c_{1} c_{2}\right\rceil}:\left(A_{1} \times A_{2}\right)^{n} \rightarrow A_{1} \times A_{2} \mid\left\lceil c_{1} c_{2}\right\rceil \in \Sigma_{\lceil 12\rceil n}\right\}_{n \in \mathbb{N}}\right)
$$

with

$$
\underline{\left\lceil c_{1} c_{2}\right\rceil}\left(\left(a_{1}, b_{1}\right), \ldots,\left(a_{n}, b_{n}\right)\right)=\left(\underline{c_{1}}\left(a_{1}, \ldots, a_{n}\right), \underline{c_{2}}\left(b_{1}, \ldots, b_{n}\right)\right) .
$$

We refer to $\mathcal{M}_{\lceil 12\rceil}$ as being the product semantics for the meet-combination of constructors of $\left\lceil\mathcal{L}_{1} \mathcal{L}_{2}\right\rceil$. In the sequel, we use $\Vdash_{\lceil 12\rceil}$ and $\vDash_{\lceil 12\rceil}$ for satisfaction and entailment in $\left\lceil\mathcal{L}_{1} \mathcal{L}_{2}\right\rceil$. Furthermore, given $M_{1} \times M_{2} \in \mathcal{M}_{\lceil 12\rceil}$ and an assignment $\rho: \Xi \rightarrow A_{1} \times A_{2}$ over $M_{1} \times M_{2}$, we denote by $\rho_{1}$ and $\rho_{2}$ the unique assignments over $M_{1}$ and $M_{2}$, respectively, such that $\rho(\xi)=\left(\rho_{1}(\xi), \rho_{2}(\xi)\right)$. 


\section{Forgetting constructors}

In practice, when meet-combining two suitable logics $\mathcal{L}_{1}$ and $\mathcal{L}_{2}$ one may want to restrict the language of the combination by dropping some constructors. More concretely, instead of working with the full signature $\Sigma_{\lceil 12\rceil}$, one may want to work with some $\Sigma \hookrightarrow \Sigma_{\lceil 12\rceil}$. The restricted language and calculus are obvious. For semantics take the reduct of each matrix in $\mathcal{M}_{\lceil 12\rceil}$.

In the next section, the results are stated and proved for the full meetcombination. Forgetting some non-inherited constructors does not disturb any of the results. In Section 5 we return to this issue.

\section{Main results}

Assuming that we are given two suitable logics $\mathcal{L}_{1}=\left(\Sigma_{1}, \Delta_{1}, \mathcal{M}_{1}\right)$ and $\mathcal{L}_{2}=$ $\left(\Sigma_{2}, \Delta_{2}, \mathcal{M}_{2}\right)$ we proceed to investigate which properties of those logics are transferred to their meet-combination $\left\lceil\mathcal{L}_{1} \mathcal{L}_{2}\right\rceil=\left(\Sigma_{\lceil 12\rceil}, \Delta_{\lceil 12\rceil}, \mathcal{M}_{\lceil 12\rceil}\right)$.

\section{Theorem 4.1 (Preservation of suitability)}

The logic $\left\lceil\mathcal{L}_{1} \mathcal{L}_{2}\right\rceil$ is suitable.

Proof: Clearly, $\left\lceil\mathrm{t}_{1} \mathrm{t}_{2}\right\rceil$ fulfills assumption (i). Moreover, $\left\lceil\mathrm{ff}_{1} \mathrm{ff}_{2}\right\rceil$ fulfills assumption (ii).

This trivial result is nonetheless useful since it allows us to iterate the process of meet-combining logics (required to be suitable). Towards establishing the preservation of soundness we need several auxiliary results.

Proposition 4.2 Let $\varphi \in L_{1}(\Xi) \cup L_{2}(\Xi), M_{1} \in \mathcal{M}_{1}, M_{2} \in \mathcal{M}_{2}$ and $\rho$ an assignment over $M_{1} \times M_{2}$. Then

$$
\llbracket \varphi \rrbracket_{\mathfrak{A}_{1} \times \mathfrak{A}_{2}, \rho}= \begin{cases}\left(\rho_{1}(\xi), \rho_{2}(\xi)\right) & \text { if } \varphi \text { is } \xi \\ \left(\llbracket \varphi \rrbracket_{\mathfrak{A}_{1}, \rho_{1}}, \llbracket \mathrm{t}_{2} \rrbracket_{\mathfrak{A}_{2}}\right) & \text { if } \varphi \text { is in } L_{1}(\Xi) \backslash \Xi \\ \left(\llbracket \mathrm{t}_{1} \rrbracket_{\mathfrak{A}_{1}}, \llbracket \varphi \rrbracket_{\mathfrak{A}_{2}, \rho_{2}}\right) & \text { if } \varphi \text { is in } L_{2}(\Xi) \backslash \Xi .\end{cases}
$$

Proof: For each $k=1,2$, the proof is carried out by a straightforward induction on $\varphi \in L_{k}(\Xi)$.

QED

Proposition 4.3 Let $\varphi \in L_{\lceil 12\rceil}(\Xi), M_{1} \in \mathcal{M}_{1}, M_{2} \in \mathcal{M}_{2}$ and $\rho$ an assignment over $M_{1} \times M_{2}$. Then

$$
\llbracket \varphi \rrbracket_{\mathfrak{A}_{1} \times \mathfrak{A}_{2}, \rho}=\left(\left(\left.\llbracket \varphi\right|_{1} \rrbracket_{\mathfrak{A}_{1} \times \mathfrak{A}_{2}, \rho}\right)_{1},\left(\left.\llbracket \varphi\right|_{2} \rrbracket_{\mathfrak{A}_{1} \times \mathfrak{A}_{2}, \rho}\right)_{2}\right) .
$$

Proof: The proof is straightforward by induction on $\varphi$.

Proposition 4.4 Let $\varphi \in L_{\lceil 12\rceil}(\Xi), M_{1} \in \mathcal{M}_{1}, M_{2} \in \mathcal{M}_{2}$ and $\rho$ an assignment over $M_{1} \times M_{2}$. Then

$$
M_{1} \times M_{2}, \rho \Vdash_{\lceil 12\rceil} \varphi \quad \text { iff } \quad M_{1},\left.\rho_{1} \Vdash_{1} \varphi\right|_{1} \text { and } M_{2},\left.\rho_{2} \Vdash_{2} \varphi\right|_{2} .
$$


Proof: Note that $M_{1} \times M_{2}, \rho \Vdash_{\lceil 12\rceil} \varphi$ iff $\llbracket \varphi \rrbracket_{\mathfrak{A}_{1} \times \mathfrak{A}_{2}, \rho}$ in $D_{1} \times D_{2}$ iff $\left(\left.\llbracket \varphi\right|_{1} \rrbracket_{\mathfrak{A}_{1} \times \mathfrak{A}_{2}, \rho}\right)_{1}$ in $D_{1},\left(\left.\llbracket \varphi\right|_{2} \rrbracket_{\mathfrak{A}_{1} \times \mathfrak{A}_{2}, \rho}\right)_{2}$ in $D_{2}$, by Proposition 4.3, iff $\left.\llbracket \varphi\right|_{1} \rrbracket_{\mathfrak{A}_{1}, \rho_{1}}$ in $D_{1},\left.\llbracket \varphi\right|_{2} \rrbracket_{\mathfrak{A}_{2}, \rho_{2}}$ in $D_{2}$, by Proposition 4.2, iff $M_{1},\left.\rho_{1} \Vdash_{1} \varphi\right|_{1}$ and $M_{2},\left.\rho_{2} \Vdash_{2} \varphi\right|_{2}$.

QED

Proposition 4.5 The lifting rule LFT is sound in $\left\lceil\mathcal{L}_{1} \mathcal{L}_{2}\right\rceil$.

Proof: Assume that $M_{1} \times M_{2},\left.\rho \Vdash_{\lceil 12\rceil} \varphi\right|_{1}$ and that $M_{1} \times M_{2},\left.\rho \Vdash_{\lceil 12\rceil} \varphi\right|_{2}$. That is, $\left.\llbracket \varphi\right|_{1} \rrbracket_{\mathfrak{A}_{1} \times \mathfrak{A}_{2}, \rho}=\left.\llbracket \varphi\right|_{2} \rrbracket_{\mathfrak{A}_{1} \times \mathfrak{A}_{2}, \rho}$ in $D_{1} \times D_{2}$. Hence, by Proposition 4.3, $\llbracket \varphi \rrbracket_{\mathfrak{A}_{1} \times \mathfrak{A}_{2}, \rho}$ in $D_{1} \times D_{2}$ and so $M_{1} \times M_{2}, \rho \Vdash_{\lceil 12\rceil} \varphi$.

QED

Proposition 4.6 The co-lifting rule cLFT is sound in $\left\lceil\mathcal{L}_{1} \mathcal{L}_{2}\right\rceil$.

Proof: Assume that $M_{1} \times M_{2}, \rho \Vdash_{\lceil 12\rceil} \varphi$. Then $\llbracket \varphi \rrbracket_{\mathfrak{A}_{1} \times \mathfrak{A}_{2}, \rho}$ in $D_{1} \times D_{2}$ and so, by Proposition $4.3,\left(\left.\llbracket \varphi\right|_{1} \rrbracket_{\mathfrak{A}_{1} \times \mathfrak{A}_{2}, \rho}\right)_{1}$ in $D_{1}$ and $\left(\left.\llbracket \varphi\right|_{2} \rrbracket_{\mathfrak{A}_{1} \times \mathfrak{A}_{2}, \rho}\right)_{2}$ in $D_{2}$. On the other hand, by Proposition 4.2, $\left(\left.\llbracket \varphi\right|_{1} \rrbracket_{\mathfrak{A}_{1} \times \mathfrak{A}_{2}, \rho}\right)_{2}$ in $D_{2}$ and $\left(\left.\llbracket \varphi\right|_{2} \rrbracket_{\mathfrak{A}_{1} \times \mathfrak{A}_{2}, \rho}\right)_{1}$ in $D_{1}$. Thus, $M_{1} \times M_{2},\left.\rho \Vdash_{\lceil 12\rceil} \varphi\right|_{1}$ and that $M_{1} \times M_{2},\left.\rho \Vdash_{\lceil 12\rceil} \varphi\right|_{2}$.

QED

Proposition 4.7 For each $k=1,2$, a sound rule in $\mathcal{L}_{k}$ is also sound in $\left\lceil\mathcal{L}_{1} \mathcal{L}_{2}\right\rceil$ provided that its conclusion is not a schema variable.

Proof: Let $r=\left(\left\{\alpha_{1}, \ldots, \alpha_{m}\right\}, \beta\right)$ be a rule in $\mathcal{L}_{1}$. Assume that $M_{1} \times M_{2}, \rho \Vdash_{\lceil 12\rceil}$ $\alpha_{j}$ for $j=1, \ldots, m$. Then $\llbracket \alpha_{j} \rrbracket_{\mathfrak{A}_{1} \times \mathfrak{A}_{2}, \rho}$ in $D_{1} \times D_{2}$ for $j=1, \ldots, m$. Hence, by Proposition $4.2, \llbracket \alpha_{j} \rrbracket_{\mathfrak{A}_{1}, \rho_{1}}$ in $D_{1}$ for $j=1, \ldots, m$ and so, by the soundness of $r$ in $\mathcal{L}_{1}$, we conclude that $\llbracket \beta \rrbracket_{\mathfrak{A}_{1}, \rho_{1}}$ in $D_{1}$. Observe that $\beta$ is not a schema variable and so, by the same proposition, $\llbracket \beta \rrbracket_{\mathfrak{A}_{1} \times \mathfrak{A}_{2}, \rho}$ in $D_{1} \times D_{2}$. Thus $M_{1} \times M_{2}, \rho \Vdash_{\lceil 12\rceil} \beta$. The same holds when $r$ in $\mathcal{L}_{2}$.

QED

Proposition 4.8 For each $k=1,2$, if $r$ is a sound rule in $\mathcal{L}_{k}$ whose conclusion is a schema variable, then, for each $c \in \Sigma_{k}, r_{c}$ is also sound in $\left\lceil\mathcal{L}_{1} \mathcal{L}_{2}\right\rceil$.

Proof: Let $r=\left(\left\{\alpha_{1}, \ldots, \alpha_{m}\right\}, \beta\right)$ be a rule in $\mathcal{L}_{1}$ where $\beta$ is a schema variable and $c$ is a constructor in $\Sigma_{1}$. Assume that $M_{1} \times M_{2},\left.\rho \Vdash_{\lceil 12\rceil} \alpha_{j}\right|_{\beta_{c}} ^{\beta}$ for $j=$ $1, \ldots, m$. Then $\left.\llbracket \alpha_{j}\right|_{\beta_{c}} ^{\beta} \rrbracket_{\mathfrak{A}_{1} \times \mathfrak{A}_{2}, \rho}$ is in $D_{1} \times D_{2}$ for $j=1, \ldots, m$. Hence, by Proposition 4.2, $\left.\llbracket \alpha_{j}\right|_{\beta_{c}} ^{\beta} \rrbracket_{\mathfrak{A}_{1}, \rho_{1}}$ is in $D_{1}$ for $j=1, \ldots, m$. On the other hand, by the soundness of $r$ in $\mathcal{L}_{1},\left\{\alpha_{1}, \ldots, \alpha_{m}\right\} \vDash_{1} \beta$. Hence, by Proposition 2.2, $\left\{\left.\alpha_{1}\right|_{\beta_{c}} ^{\beta}, \ldots,\left.\alpha_{m}\right|_{\beta_{c}} ^{\beta}\right\} \vDash_{1} \beta_{c}$. Therefore, $\llbracket \beta_{c} \rrbracket_{\mathfrak{A}_{1}, \rho_{1}}$ is in $D_{1}$. Observe that $\beta_{c}$ is not a schema variable and so, by Proposition $4.2, \llbracket \beta_{c} \rrbracket_{\mathfrak{A}_{1} \times \mathfrak{A}_{2}, \rho}$ in $D_{1} \times D_{2}$. Thus $M_{1} \times M_{2}, \rho \Vdash_{\lceil 12\rceil} \beta_{c}$. The same holds when $r$ in $\mathcal{L}_{2}$.

QED

\section{Theorem 4.9 (Preservation of soundness)}

If $\mathcal{L}_{1}$ and $\mathcal{L}_{2}$ are sound then $\left\lceil\mathcal{L}_{1} \mathcal{L}_{2}\right\rceil$ is sound.

Proof: Assume that $\mathcal{L}_{1}$ and $\mathcal{L}_{2}$ are sound. Then, in particular, all the rules in $\mathcal{L}_{1}$ and in $\mathcal{L}_{2}$ whose conclusion is not a schema variable are sound in $\left\lceil\mathcal{L}_{1} \mathcal{L}_{2}\right\rceil$ by Proposition 4.7. Furthermore, $r_{c}$ is also sound, by Proposition 4.8, for each rule $r \in \mathcal{L}_{k}$ whose conclusion is a schema variable and $c$ is a constructor in $\Sigma_{k}$. Moreover, the rules LFT and cLFT are sound thanks to Proposition 4.5 and Proposition 4.6, respectively. Therefore, $\left\lceil\mathcal{L}_{1} \mathcal{L}_{2}\right\rceil$ is sound taking into account that $\vDash_{\lceil 12\rceil}$ is closed for substitution.

QED 
The task now is to show that if $\mathcal{L}_{1}$ and $\mathcal{L}_{2}$ are complete then so is $\left\lceil\mathcal{L}_{1} \mathcal{L}_{2}\right\rceil$. However, we are able to establish this result only for concrete formulas (Theorem 4.15). Thus, it becomes handy to say that a logic is concretely complete if it is complete with respect to concrete formulas. We start by proving the relevant lemmas.

Proposition 4.10 For each $k=1,2$ and $\Gamma \cup\{\varphi\} \subseteq L_{k}$, if $\Gamma \vdash_{k} \varphi$ then $\Gamma \vdash_{\lceil 12\rceil} \varphi$.

Proof: We start by showing that if $\Gamma \vdash_{k} \varphi$ then there is a derivation of $\varphi$ from $\Gamma$ composed by concrete formulas in $L_{k}$. The proof follows by induction on a derivation of $\varphi$ from $\Gamma$.

(1) $\varphi \in \Gamma$. Straightforward since $\varphi$ is concrete.

(2) $\varphi$ is an instance of an axiom. Straightforward since $\varphi$ is concrete.

(3) $\varphi$ is an instance of $\left(\left\{\alpha_{1}, \ldots, \alpha_{m}\right\}, \beta\right)$ using substitution $\sigma$. Then $\Gamma \vdash_{k} \sigma\left(\alpha_{j}\right)$ for $j=1, \ldots, m$ and so $\Gamma \vdash_{k} \sigma^{\prime}\left(\sigma\left(\alpha_{j}\right)\right)$, with a derivation with the same number of steps, where $\sigma^{\prime}$ is such that $\sigma^{\prime}(\xi)$ is a concrete formula for every $\xi$. Therefore, by the induction hypothesis, there is a derivation of $\sigma^{\prime}\left(\sigma\left(\alpha_{j}\right)\right)$ from $\Gamma$ composed by concrete formulas for $j=1, \ldots, m$. Thus, using the same rule, we conclude that $\Gamma \vdash_{k} \sigma(\beta)$ since $\sigma^{\prime}(\sigma(\beta))=\sigma(\beta)$.

The thesis follows since a derivation composed by concrete formulas in $\mathcal{L}_{k}$ is also a derivation in $\left\lceil\mathcal{L}_{1} \mathcal{L}_{2}\right\rceil$.

QED

Proposition 4.11 If $\mathcal{L}_{1}$ and $\mathcal{L}_{2}$ are concretely complete then

$$
\text { if } \Gamma \forall_{\lceil 12\rceil} \varphi \text { then } \Gamma \not \nvdash_{\lceil 12\rceil} \varphi
$$

for every $\Gamma \cup\{\varphi\} \subset L_{k}$ with $k=1,2$.

Proof: Assume that $k=1$ and that $\Gamma \nvdash_{\lceil 12\rceil} \varphi$. Then, by Proposition 4.10, $\Gamma \nvdash_{1} \varphi$ and, since $\mathcal{L}_{1}$ is concretely complete then $\Gamma \forall_{1} \varphi$. That is, there is $M_{1} \in \mathcal{M}_{1}$ such that $M_{1} \Vdash_{1} \gamma$ for every $\gamma \in \Gamma$ and $M_{1} \forall{ }_{1} \varphi$. Hence, $\left.M_{1} \Vdash_{1} \gamma\right|_{1}$ for every $\gamma \in \Gamma$ and $\left.M_{1} \forall_{1} \varphi\right|_{1}$. Choose $M_{2} \in \mathcal{M}_{2}$. Then $\left.M_{2} \Vdash_{2} \gamma\right|_{2}$ for every $\gamma \in \Gamma$ and $\left.M_{2} \Vdash_{2} \varphi\right|_{2}$. Hence, by Proposition 4.4, $M_{1} \times M_{2} \Vdash_{\lceil 12\rceil} \gamma$ for every $\gamma \in \Gamma$ and $M_{1} \times M_{2} \forall_{\lceil 12\rceil} \varphi$. Therefore, $\Gamma \forall_{\lceil 12\rceil} \varphi$.

QED

Proposition 4.12 For every $\varphi \in L_{\lceil 12\rceil}, \mathrm{ff}_{k} \vdash_{\lceil 12\rceil} \varphi$.

Proof: Indeed, using rule FX, $\mathrm{ff}_{1} \vdash_{\lceil 12\rceil} \mathrm{ff}_{2}$. On the other hand, $\left.\mathrm{ff}_{1} \vdash_{\lceil 12\rceil} \varphi\right|_{1}$ and $\left.\mathrm{ff}_{2} \vdash_{\lceil 12\rceil} \varphi\right|_{2}$. Hence, the thesis follows by the lifting rule LFT. QED

Proposition 4.13 Assume that

$$
\text { if } \Gamma^{\prime} \forall_{\lceil 12\rceil} \varphi^{\prime} \text { then } \Gamma^{\prime} \forall_{\lceil 12\rceil} \varphi^{\prime}
$$

for every $\Gamma^{\prime} \cup\left\{\varphi^{\prime}\right\} \subset L_{k}$ for $k=1,2$. Then, for every $\Gamma \subset L_{1} \cup L_{2}$ and $\varphi \in L_{\lceil 12\rceil}$,

$$
\text { if } \Gamma \forall_{\lceil 12\rceil} \varphi \text { then } \Gamma \forall_{\lceil 12\rceil} \varphi \text {. }
$$


Proof: Let $\Gamma=\Gamma_{1} \cup \Gamma_{2}$ where $\Gamma_{1} \subseteq L_{1}$ and $\Gamma_{2} \subseteq L_{2}$. We consider three cases. (1) $\Gamma_{1}$ does not have a model in $\mathcal{L}_{1}$. Assume $\Gamma \vDash_{\lceil 12\rceil} \varphi$. Observe that $\Gamma_{1} \vDash_{1} \mathrm{ff}_{1}$. Hence, by completeness of $\mathcal{L}_{1}, \Gamma_{1} \vdash_{1} \mathrm{ff}_{1}$ and so $\Gamma_{1} \vdash_{\lceil 12\rceil} \mathrm{ff}_{1}$, by Proposition 4.10. Hence, by Proposition 4.12, $\Gamma_{1} \vdash_{\lceil 12\rceil} \varphi$ and so $\Gamma \vdash_{\lceil 12\rceil} \varphi$.

(2) $\Gamma_{2}$ does not have a model in $\mathcal{L}_{2}$. Similar to (1).

(3) $\Gamma_{1}$ and $\Gamma_{2}$ have models in $\mathcal{L}_{1}$ and $\mathcal{L}_{2}$, respectively. Assume that $\Gamma \nvdash_{\lceil 12\rceil} \varphi$. Then, taking into account the lifting rule LFT,

$$
\text { either }\left.\Gamma \forall_{\lceil 12\rceil} \varphi\right|_{1} \text { or }\left.\Gamma \forall_{\lceil 12\rceil} \varphi\right|_{2} .
$$

Consider two cases.

(a) Assume that the main constructor in $\varphi$ is in $\Sigma_{1}$. Hence, $\left.\Gamma \forall_{\lceil 12\rceil} \varphi\right|_{1}$ and so $\left.\Gamma_{1} \forall_{\lceil 12\rceil} \varphi\right|_{1}$. Thus, by hypothesis, $\left.\Gamma_{1} \forall_{\lceil 12\rceil} \varphi\right|_{1}$. Hence, there is $M_{1}$ such that $M_{1} \Vdash_{1} \gamma_{1}$ for every $\gamma_{1} \in \Gamma_{1}$ and $\left.M_{1} \forall \nvdash_{1} \varphi\right|_{1}$. Let $M_{2}$ be a model of $\Gamma_{2}$ in $\mathcal{L}_{2}$. Then $M_{1} \times M_{2} \Vdash_{\lceil 12\rceil} \gamma$ for every $\gamma \in \Gamma_{1} \cup \Gamma_{2}$ and $M_{1} \times M_{2} \forall_{\lceil 12\rceil} \varphi$. That is, $\Gamma \not \curlyvee 12\rceil_{\varphi}$.

(b) Assume that the main constructor in $\varphi$ is in $\Sigma_{2}$. Similar to (a). QED

Proposition 4.14 Assume that

$$
\text { if } \Gamma^{\prime} \forall_{\lceil 12\rceil} \varphi^{\prime} \text { then } \Gamma^{\prime} \forall_{\lceil 12\rceil} \varphi^{\prime}
$$

for every $\Gamma^{\prime} \subset L_{1} \cup L_{2}$ and $\varphi^{\prime} \in L_{\lceil 12\rceil}$. Then

$$
\text { if } \Gamma \forall_{\lceil 12\rceil} \varphi \text { then } \Gamma \forall_{\lceil 12\rceil} \varphi
$$

for every $\Gamma \cup\{\varphi\} \subset L_{\lceil 12\rceil}$.

Proof: Assume that $\Gamma \cup\{\varphi\} \in L_{\lceil 12\rceil}$ and $\Gamma \nvdash_{\lceil 12\rceil} \varphi$. Then

$$
\left.\left.\Gamma\right|_{1} \cup \Gamma\right|_{2} \nvdash_{\lceil 12\rceil} \varphi
$$

taking into account the co-lifting rule cLFT, where $\left.\Gamma\right|_{k}=\left\{\left.\gamma\right|_{k}: \gamma \in \Gamma\right\}$ for $k=1,2$. Since no combined constructors occur in both $\left.\Gamma\right|_{1}$ and $\left.\Gamma\right|_{2}$, we can use the hypothesis to conclude that

$$
\left.\left.\Gamma\right|_{1} \cup \Gamma\right|_{2} \not \nvdash_{\lceil 12\rceil} \varphi \text {. }
$$

That is, there are $M_{1} \in \mathcal{M}_{1}$ and $M_{2} \in \mathcal{M}_{2}$ such that

$$
M_{1} \times M_{2} \Vdash_{\lceil 12\rceil} \gamma^{\prime} \text {, for every }\left.\left.\gamma^{\prime} \in \Gamma\right|_{1} \cup \Gamma\right|_{2} \text { and } M_{1} \times M_{2} \forall_{\lceil 12\rceil} \varphi .
$$

Hence, $\llbracket \gamma^{\prime} \rrbracket_{\mathfrak{A}_{1} \times \mathfrak{A}_{2}} \in D_{1} \times D_{2}$ for every $\left.\left.\gamma^{\prime} \in \Gamma\right|_{1} \cup \Gamma\right|_{2}$ and $\llbracket \varphi \rrbracket_{\mathfrak{A}_{1} \times \mathfrak{A}_{2}} \notin D_{1} \times D_{2}$. Therefore,

$$
\left(\llbracket \gamma^{\prime} \rrbracket_{\mathfrak{A}_{1} \times \mathfrak{A}_{2}}\right)_{1} \in D_{1} \quad \text { and } \quad\left(\llbracket \gamma^{\prime} \rrbracket_{\mathfrak{A}_{1} \times \mathfrak{A}_{2}}\right)_{2} \in D_{2}
$$

for every $\left.\left.\gamma^{\prime} \in \Gamma\right|_{1} \cup \Gamma\right|_{2}$. Let $\gamma \in \Gamma$. By Proposition 4.3,

$$
\llbracket \gamma \rrbracket_{\mathfrak{A}_{1} \times \mathfrak{A}_{2}}=\left(\left(\left.\llbracket \gamma\right|_{1} \rrbracket_{\mathfrak{A}_{1} \times \mathfrak{A}_{2}}\right)_{1},\left(\left.\llbracket \gamma\right|_{2} \rrbracket_{\mathfrak{A}_{1} \times \mathfrak{A}_{2}}\right)_{2}\right) .
$$

Thus, $\llbracket \gamma \rrbracket_{\mathfrak{A}_{1} \times \mathfrak{A}_{2}} \in D_{1} \times D_{2}$ and so $M_{1} \times M_{2} \Vdash \Gamma$. Therefore, $\Gamma \not_{\lceil 12\rceil} \varphi$. QED 


\section{Theorem 4.15 (Preservation of concrete completeness)}

If $\mathcal{L}_{1}$ and $\mathcal{L}_{2}$ are concretely complete then $\left\lceil\mathcal{L}_{1} \mathcal{L}_{2}\right\rceil$ is concretely complete.

Proof: Assume that $\mathcal{L}_{1}$ and $\mathcal{L}_{2}$ are concretely complete. Then, by Proposition 4.11,

$$
\text { if } \Gamma \forall_{\lceil 12\rceil} \varphi \text { then } \Gamma \forall_{\lceil 12\rceil} \varphi
$$

for every $\Gamma \cup\{\varphi\} \subset L_{k}$ for $k=1,2$. Hence, for every $\Gamma \subset L_{1} \cup L_{2}$ and $\varphi \in L_{\lceil 12\rceil}$,

$$
\text { if } \Gamma \forall_{\lceil 12\rceil} \varphi \text { then } \Gamma \not_{\lceil 12\rceil} \varphi
$$

using Proposition 4.13. Thus, thanks to Proposition 4.14,

$$
\text { if } \Gamma \forall_{\lceil 12\rceil} \varphi \text { then } \Gamma \forall_{\lceil 12\rceil} \varphi
$$

for every $\Gamma \cup\{\varphi\} \in L_{\lceil 12\rceil}$.

The completeness result was established only for concrete formulas since it depends on Proposition 4.11 and, so, on Proposition 4.10. Recall that the latter states that a concrete derivation in each $\mathcal{L}_{k}$ can be ported to $\left\lceil\mathcal{L}_{1} \mathcal{L}_{2}\right\rceil$. This result does not hold for schematic formulas because in $\left\lceil\mathcal{L}_{1} \mathcal{L}_{2}\right\rceil$ we only have the tagged versions of the liberal rules in $\mathcal{L}_{k}$.

Finally, we turn our attention to the preservation of consistency. To this end, we check first if $\left\lceil\mathcal{L}_{1} \mathcal{L}_{2}\right\rceil$ is a conservative extension of $\mathcal{L}_{1}$ and $\mathcal{L}_{2}$.

\section{Theorem 4.16 (Concrete extensiveness)}

For each $k=1,2$ and $\Gamma \cup\{\varphi\} \subset L_{k}$,

$$
\text { if } \Gamma \vDash_{k} \varphi \text { then } \Gamma \vDash_{\lceil 12\rceil} \varphi \text {. }
$$

Proof: Without loss of generality, let $\Gamma \cup\{\varphi\} \subset L_{1}$. Assume that $\Gamma \vDash_{1} \varphi$. Let $M_{1} \in \mathcal{M}_{1}$ and $M_{2} \in \mathcal{M}_{2}$ be such that $M_{1} \times M_{2} \Vdash_{\lceil 12\rceil} \gamma$ for each $\gamma \in \Gamma$. Then, by Proposition 4.4, $M_{1} \Vdash_{1} \gamma$ for each $\gamma \in \Gamma$. Then, by the hypothesis, $M_{1} \Vdash_{1}$ $\varphi$. Moreover, $M_{2} \Vdash_{2} \mathbb{t}^{(n)}$ for each $n \in \mathbb{N}$. Hence, by the same proposition, $M_{1} \times M_{2} \Vdash_{\lceil 12\rceil} \varphi$.

QED

Remark that $\left\lceil\mathcal{L}_{1} \mathcal{L}_{2}\right\rceil$ is an extension of $\mathcal{L}_{1}$ and $\mathcal{L}_{2}$ only for concrete formulas. Indeed, from $M_{1}, \rho_{1} \Vdash_{1} \xi$ we would not be able to infer $M_{1} \times M_{2}, \rho \Vdash_{\lceil 12\rceil} \xi$, since we would not know if $M_{2}, \rho_{2} \Vdash_{2} \xi$ or not. On the other hand, the conservative nature of the two extensions holds also for schema formulas:

Theorem 4.17 (Conservativeness)

For each $k=1,2$ and $\Gamma \cup\{\varphi\} \subset L_{k}(\Xi)$,

$$
\text { if } \Gamma \vDash_{\lceil 12\rceil} \varphi \text { then } \Gamma \vDash_{k} \varphi \text {. }
$$

Proof: Without loss of generality, let $\Gamma \cup\{\varphi\} \subset L_{1}(\Xi)$. Assume that $\Gamma \vDash_{\lceil 12\rceil} \varphi$ and let $M_{1} \in \mathcal{M}_{1}$ and $\rho_{1}$ an assignment over $M_{1}$ such that $M_{1}, \rho_{1} \Vdash_{1} \Gamma$. Hence $\llbracket \gamma \rrbracket_{\mathfrak{A}_{1} \rho_{1}} \in D_{1}$ for every $\gamma \in \Gamma$. Let $M_{2} \in \mathcal{M}_{2}$. Denote by $\rho$ the unique assignment over $M_{1} \times M_{2}$ such that $(\rho)_{1}=\rho_{1}$ and $(\rho)_{2}(\xi)=\llbracket \mathbb{t} \rrbracket_{\mathfrak{A}_{2}}$. Then, by Proposition 4.2, $\llbracket \gamma \rrbracket_{\mathfrak{A}_{1} \times \mathfrak{A}_{2} \rho} \in D_{1} \times D_{2}$ for every $\gamma \in \Gamma$. Therefore, $\llbracket \varphi \rrbracket_{\mathfrak{A}_{1} \times \mathfrak{A}_{2} \rho} \in$ $D_{1} \times D_{2}$ and so by the same proposition, $\llbracket \varphi \rrbracket_{\mathfrak{A}_{1} \rho_{1}} \in D_{1}$. That is, $M_{1}, \rho_{1} \Vdash \varphi$ and so $\Gamma \vDash_{1} \varphi$.

QED 
It is worthwhile to mention again that, thanks to the conservativeness result above, cLFT guarantees that each meet-combined constructor enjoys only the common properties of its components in the original logic. As an immediate corollary, we obtain:

\section{Theorem 4.18 (Preservation of consistency)}

If $\mathcal{L}_{1}$ and $\mathcal{L}_{2}$ are consistent then so is $\left\lceil\mathcal{L}_{1} \mathcal{L}_{2}\right\rceil$.

\section{$5 \quad$ Worked examples}

Our objective now is to illustrate the proposed way of meet-combining suitable logics. We also provide a counter-example concerning the unsoundness of nontagged liberal rules.

\section{Meet-combination of classical and intuitionistic logics}

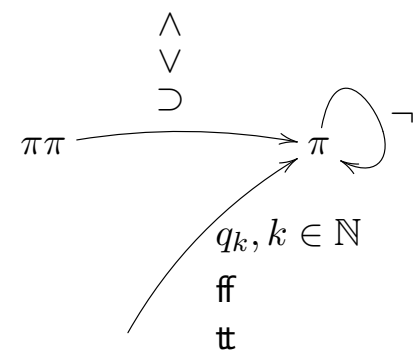

Figure 1: Propositional signature.

Let $\mathrm{CPL}=\left(\Sigma_{\mathrm{C}}, \Delta_{\mathrm{C}}, \mathcal{M}_{\mathrm{C}}\right)$ be classical propositional logic. More concretely, let:

- $\Sigma_{\mathrm{C}}$ be a clone of the signature in Figure 1 containing $\neg_{\mathrm{C}}, \wedge_{\mathrm{C}}$, etc.

- $\Delta_{\mathrm{C}}$ contains the tautologies as axioms plus modus ponens:

$$
\frac{\xi_{1}\left(\xi_{1} \supset_{\mathrm{C}} \xi_{2}\right)}{\xi_{2}} \quad\left(\mathrm{MP}_{\mathrm{C}}\right) \text {. }
$$

- $\mathcal{M}_{\mathrm{C}}$ is composed of the matrices induced by valuations. Recall that each valuation $v:\left\{q_{\mathrm{C} k}: k \in \mathbb{N}\right\} \rightarrow\{0,1\}$ induces a matrix $M_{v}$ with $A_{v}=\{0,1\}$ satisfying precisely the same formulas for each assignment.

Let IPL $=\left(\Sigma_{1}, \Delta_{1}, \mathcal{M}_{\mathrm{l}}\right)$ be intuitionistic propositional logic. More concretely, let:

- $\Sigma_{\mathrm{I}}$ be a clone of the signature in Figure 1 containing $\neg_{\mathrm{I}}, \wedge_{\mathrm{I}}$, etc.

- $\Delta_{I}$ contains the usual axioms of intuitionistic logic (see, for instance, [9]) plus modus ponens:

$$
\frac{\xi_{1}\left(\xi_{1} \supset_{\mathrm{I}} \xi_{2}\right)}{\xi_{2}} \quad\left(\mathrm{MP}_{\mathrm{I}}\right)
$$


- $\mathcal{M}_{1}$ is composed of the matrices induced by Heyting algebras. Recall that each Heyting algebra $\mathfrak{H}$ together with its $T$ as the unique distinguished value induces a matrix $M_{\mathfrak{H}}$ satisfying precisely the same formulas for each assignment.

Thanks to the results in Section 4, the meet-combination

$$
\mathrm{CIPL}=\lceil\mathrm{CPLIPL}\rceil=\left(\Sigma_{\mathrm{Cl}}, \Delta_{\mathrm{Cl}}, \mathcal{M}_{\mathrm{Cl}}\right)
$$

of these two logics is sound and (concretely) complete. Furthermore, it is a conservative (concretely) extension of each of them and, so, consistent. We now proceed to study this meet-combination (that we may call classical-intuitionistic propositional logic) in more detail.

Observe that in $\Sigma_{\mathrm{Cl}}$ we find three negations:

$$
\begin{aligned}
\neg \mathrm{C} & =\left\lceil\neg \mathrm{C} \mathrm{t}_{\mid}^{(1)}\right\rceil \\
\neg \mathbf{I} & =\left\lceil\mathrm{t}_{\mathrm{C}}^{(1)} \neg \mathbf{I}\right\rceil \\
\neg \mathrm{Cl} & =\lceil\neg \mathrm{C} \neg \mathbf{\neg}\rceil .
\end{aligned}
$$

The same goes for the other connectives.

Given the conservative nature of the embeddings

$$
\begin{aligned}
& \eta_{\mathrm{C}}: \mathrm{CPL} \rightarrow \mathcal{L} \\
& \eta_{\mathrm{I}}: \mathrm{IPL} \rightarrow \mathcal{L},
\end{aligned}
$$

$\neg \mathrm{C}$ behaves as in CPL within $\eta_{\mathrm{C}}(\mathrm{CPL})$ and $\neg$ behaves as in IPL within $\eta_{l}(\mathrm{IPL})$. For instance, we do have

$$
\vdash_{\mathrm{Cl}}\left(q_{\mathrm{C} 1} \vee_{\mathrm{C}}\left(\neg \mathrm{C} q_{\mathrm{C} 1}\right)\right)
$$

since middle excluded is inherited as it is (as an axiom). However,

$$
\forall \mathrm{Cl}\left(q_{11} \vee_{\mathrm{I}}\left(\neg_{\mathrm{I}} q_{11}\right)\right)
$$

since

$$
\nvdash_{\mathrm{I}}\left(q_{\mathrm{I1}} \vee_{\mathrm{I}}\left(\neg_{\mathrm{I}} q_{11}\right)\right) \text {. }
$$

It should be stressed that $\neg \mathrm{C}$ only behaves classically within $\eta_{\mathrm{C}}(\mathrm{CPL})$. Beyond that, things get more complicated since we can not invoke the conservative nature of the embedding. For example, we still get

$$
\text { (†) }\left(q_{\mathrm{C} 1} \vee_{\mathrm{I}}\left(\neg \mathrm{C} q_{\mathrm{C} 1}\right)\right)
$$

as a theorem in the meet-combination, but

( $) \quad\left(\left(\neg \mathrm{C}\left(\neg \mathrm{C} q_{11}\right)\right) \supset_{\mathrm{l}} q_{11}\right)$ 
is not a theorem since it is not valid. Indeed, the following derivation establishes the unexpected hybrid middle excluded $(\dagger)$

$\begin{array}{llr}1 & \left(q_{\mathrm{C} 1} \mathbb{t}_{C}^{(2)}\left(\neg \mathrm{C} q_{\mathrm{C} 1}\right)\right) & \mathrm{AX}_{\mathrm{C}} \\ 2 & \mathrm{t}_{\mathrm{I}} & \mathrm{AX}_{\mathbf{I}} \\ 3 & \left(\mathrm{t}_{\mathbf{I}} \supset_{\mathrm{I}}\left(\mathrm{t}_{\mathrm{I}} \vee_{\mathrm{I}}\left(\mathrm{t}_{1}^{(1)} \mathrm{t}_{\mathbf{I}}\right)\right)\right) & \mathrm{AX}_{\mathbf{I}} \\ 4 & \left(\mathrm{t}_{\mathbf{I}} \vee_{\mathrm{I}}\left(\mathrm{t}_{1}^{(1)} \mathrm{t}_{\mathbf{I}}\right)\right) & \mathrm{MP}_{\mathbf{I}} 2,3 \\ 5 & \left(q_{\mathrm{C} 1} \vee_{\mathbf{I}}\left(\neg \mathrm{C} q_{\mathrm{C} 1}\right)\right) & \text { LFT } 1,4\end{array}$

while the following matrix does not satisfy $(\ddagger)$ :

$$
M=M_{1} \times M_{2}
$$

where $M_{1}$ is any matrix of CPL and $M_{2}$ is a matrix of IPL induced by any Heyting algebra in which the denotation of $q_{\mathrm{I} 1}$ is not distinguished.

Concerning $\neg \mathrm{Cl}$, one would expect it to behave intuitionistically since it inherits only the properties common to $\neg \mathrm{C}$ and $\neg$. In order to illustrate this fact in a very simple case, observe that

$$
\vdash_{\mathrm{Cl}}(\neg \mathrm{Cl}(\neg \mathrm{Cl} \varphi)) \supset_{\mathrm{Cl}} \varphi
$$

does not hold in general. Just consider an arbitrary $\varphi \in \eta_{\mathrm{I}}\left(L_{\mathrm{I}}\right)$. Then, by cLFT we would obtain

$$
\vdash_{\mathrm{Cl}}(\neg \mathrm{I}(\neg \mathrm{I} \varphi)) \supset_{\mathrm{I}} \varphi
$$

and, so, by the conservative nature of $\eta_{\mathrm{l}}$, we would establish

$$
\vdash_{I}\left(\neg_{I}(\neg \mid \varphi)\right) \supset_{I} \varphi .
$$

On the other hand, for instance, we do have

$$
\vdash_{\mathrm{Cl}} \varphi \supset_{\mathrm{Cl}}(\neg \mathrm{Cl}(\neg \mathrm{Cl} \varphi))
$$

in general, since it is a common property of the two original negations.

The crucial difference between meet-combination and fibring [5] is clearly illustrated by this connective $\supset \mathrm{Cl}$ (the meet-combination of classical and intuitionistic implications). Here it is intuitionistic while, in fibring, sharing these two implications leads to the classical implication [6].

As expected, Peirce's Law holds within $\eta_{\mathrm{C}}(\mathrm{CPL})$ for $\supset_{\mathrm{C}}$, but not in general within CIPL for $\supset_{\mathrm{Cl}}$, since it is not a common property of $\supset_{\mathrm{C}}$ and $\supset_{\mathrm{I}}$. If Peirce's Law were to hold for $\supset_{\mathrm{Cl}}$, then by cLFT it would hold for $\supset_{\text {I }}$ within CIPL and, so, it would also hold for $\supset$, within IPL.

In $\Sigma_{\mathrm{Cl}}$ one finds also other, at first sight less useful, meet-combined constructors, e.g. the meet-combination $\left[\wedge_{c} \vee_{1}\right\rceil$ of the classical conjunction with the intuitionistic disjunction. One may wish to drop them and can do so. But they can be useful for studying the common properties of their components. For instance, $\left\lceil\wedge_{C} \vee_{1}\right\rceil$ inherits only the common properties of classical conjunction and intuitionistic disjunction. For results on such meet-combinations but only within the setting of classical logic see [7].

Before concluding this preliminary study of classical-intuitionistic propositional logic, observe that the non-tagged versions of both modus ponens rules are not sound in CIPL. Indeed, 
(i) $\xi_{1},\left(\xi_{1} \supset_{\mathrm{C}} \xi_{2}\right) \forall_{\mathrm{Cl}} \xi_{2}$;

(ii) $\xi_{1},\left(\xi_{1} \supset_{1} \xi_{2}\right) \forall_{\mathrm{Cl}} \xi_{2}$.

For (i) consider an arbitrary matrix $M=M_{1} \times M_{2} \in \mathcal{M}_{\mathrm{Cl}}$ and $\rho$ such that:

- $\rho\left(\xi_{1}\right) \in D_{1} \times D_{2}$;

- $\rho_{1}\left(\xi_{2}\right) \in D_{1}$ and $\rho_{2}\left(\xi_{2}\right) \notin D_{2}$.

Clearly, $M \rho \Vdash_{\mathrm{Cl}} \xi_{1}$ and $M \rho \Vdash_{\mathrm{Cl}}\left(\xi_{1} \supset_{\mathrm{C}} \xi_{2}\right)$, but $M \rho \forall_{\mathrm{Cl}} \xi_{2}$. A counter-example for (ii) is easily built in a similar way.

\section{Meet-combination of modal logics}

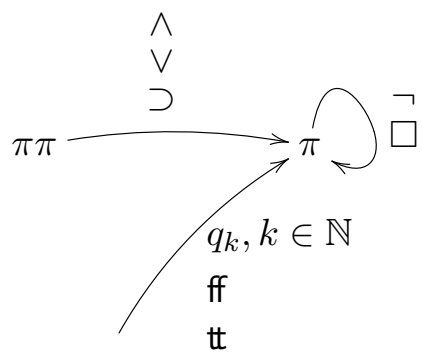

Figure 2: Modal propositional signature.

Let $\mathrm{M} 4 \mathrm{PL}=\left(\Sigma_{4}, \Delta_{4}, \mathcal{M}_{4}\right)$ be modal 4 propositional logic. More concretely, let:

- $\Sigma_{4}$ be a clone of the signature in Figure 2 containing $\square_{4}, \neg_{4}, \wedge_{4}$, etc.

- $\Delta_{4}$ contains the tautological formulas over $\Sigma_{4}$ as axioms, the normality axiom

$$
\left(\left(\square_{4}\left(\xi_{1} \supset_{4} \xi_{2}\right)\right) \supset_{4}\left(\left(\square_{4} \xi_{1}\right) \supset_{4}\left(\square_{4} \xi_{2}\right)\right)\right) \quad\left(\mathrm{NORM}_{4}\right)
$$

and the transitivity axiom

$$
\left(\left(\square_{4} \xi_{1}\right) \supset_{4}\left(\square_{4}\left(\square_{4} \xi_{1}\right)\right)\right) \quad\left(\mathrm{AX}_{4}\right),
$$

plus modus ponens

$$
\frac{\xi_{1} \quad\left(\xi_{1} \supset_{4} \xi_{2}\right)}{\xi_{2}} \quad\left(\mathrm{MP}_{4}\right)
$$

and necessitation

$$
\frac{\xi_{1}}{\left(\square_{4} \xi_{1}\right)} \quad\left(\mathrm{NEC}_{4}\right)
$$

- $\mathcal{M}_{4}$ is composed of the matrices induced by transitive Kripke structures. Recall that, in general, each Kripke structure $K=(W, R, V)$ induces a modal algebra $\mathfrak{A}_{K}$ which in turn together with set $W$ as the unique distinguished value induces a matrix $M_{K}$ satisfying precisely the same formulas for each assignment. 
Let MTPL $=\left(\Sigma_{\mathrm{T}}, \Delta_{\mathrm{T}}, \mathcal{M}_{\mathrm{T}}\right)$ be modal T propositional logic. More concretely, let:

- $\Sigma_{\mathrm{T}}$ be a clone of the signature in Figure 2 containing $\square_{\mathrm{T}}, \neg_{\mathrm{T}}, \wedge_{\mathrm{T}}$, etc.

- $\Delta_{\mathrm{T}}$ contains the tautological formulas over $\Sigma_{\mathrm{T}}$ as axioms, the normality axiom

$$
\left(\left(\square_{\mathbf{T}}\left(\xi_{1} \supset_{\mathbf{T}} \xi_{2}\right)\right) \supset_{\mathbf{T}}\left(\left(\square_{\mathbf{T}} \xi_{1}\right) \supset_{\mathbf{T}}\left(\square_{\mathbf{T}} \xi_{2}\right)\right)\right) \quad\left(\mathrm{NORM}_{\mathbf{T}}\right)
$$

and the reflexivity axiom

$$
\left(\left(\square_{\mathrm{T}} \xi_{1}\right) \supset_{\mathrm{T}} \xi_{1}\right) \quad\left(\mathrm{AX}_{\mathrm{T}}\right),
$$

plus modus ponens

$$
\frac{\xi_{1}\left(\xi_{1} \supset \mathrm{T} \xi_{2}\right)}{\xi_{2}} \quad\left(\mathrm{MP}_{\mathrm{T}}\right)
$$

and necessitation

$$
\frac{\xi_{1}}{\left(\square_{\mathrm{T}} \xi_{1}\right)} \quad\left(\mathrm{NEC}_{\mathrm{T}}\right)
$$

- $\mathcal{M}_{\mathrm{T}}$ is composed of the matrices induced by reflexive Kripke structures.

The results in Section 4 guarantee that the meet-combination

$$
\mathrm{M} 4 \mathrm{TL}=\lceil\mathrm{M} 4 \mathrm{PL} \mathrm{MTPL}\rceil=\left(\Sigma_{4 \mathrm{~T}}, \Delta_{4 \mathrm{~T}}, \mathcal{M}_{4 \mathrm{~T}}\right)
$$

of these two logics is sound and (concretely) complete. Furthermore, it is a conservative (concretely) extension of each of them and, so, consistent. We now proceed to study this meet-combination (that we may call $\lceil 4 \mathrm{~T}\rceil$-modal propositional logic) in more detail.

Observe that in this modal logic we have three modal boxes:

$$
\begin{aligned}
\square_{4} & =\left\lceil\square_{4} \mathrm{tt}_{\mathrm{T}}^{(1)}\right\rceil \\
\square_{\mathrm{T}} & =\left\lceil\mathrm{t}_{4}^{(1)} \square_{\mathrm{T}}\right\rceil \\
\square_{4 \mathrm{~T}} & =\left\lceil\square_{4} \square_{\mathrm{T}}\right\rceil .
\end{aligned}
$$

The same goes for the propositional connectives.

As expected, since

$$
\left\{\begin{array}{l}
\vdash_{4 \mathrm{~T}}\left(\left(\square_{4}\left(\xi_{1} \supset_{4} \xi_{2}\right)\right) \supset_{4}\left(\left(\square_{4} \xi_{1}\right) \supset_{4}\left(\square_{4} \xi_{2}\right)\right)\right) \\
\vdash_{4 \mathrm{~T}}\left(\left(\square_{\mathrm{T}}\left(\xi_{1} \supset_{\mathrm{T}} \xi_{2}\right)\right) \supset_{\mathrm{T}}\left(\left(\square_{\mathrm{T}} \xi_{1}\right) \supset_{\mathrm{T}}\left(\square_{\mathrm{T}} \xi_{2}\right)\right)\right),
\end{array}\right.
$$

using the lifting rule we obtain

$$
\vdash_{4 \mathrm{~T}}\left(\left(\square_{4 \mathrm{~T}}\left(\xi_{1} \supset_{4 \mathrm{~T}} \xi_{2}\right)\right) \supset_{4 \mathrm{~T}}\left(\left(\square_{4 \mathrm{~T}} \xi_{1}\right) \supset_{4 \mathrm{~T}}\left(\square_{4 \mathrm{~T}} \xi_{2}\right)\right)\right) .
$$


Actually, in the same way we also obtain, for example,

$$
\vdash_{4 \mathrm{~T}}\left(\left(\square_{4 \mathrm{~T}}\left(\xi_{1} \supset_{4} \xi_{2}\right)\right) \supset_{4}\left(\left(\square_{4 \mathrm{~T}} \xi_{1}\right) \supset_{4}\left(\square_{4 \mathrm{~T}} \xi_{2}\right)\right)\right) .
$$

Furthermore, also by lifting, we have:

$$
\xi_{1} \vdash_{4 \mathrm{~T}}\left(\square_{4 \mathrm{~T}} \xi_{1}\right) .
$$

On the other hand, contrarily to what would happen in a fibring sharing the two modal boxes (resulting in a $\mathrm{S} 4 \mathrm{box}$ ), $\square_{4 \mathrm{~T}}$ does not fulfill the transitivity and the reflexivity axioms (with respect to $\supset_{4 \mathrm{~T}}$ ). Instead it fulfills only what is common to both axioms:

$$
\vdash_{4 \mathrm{~T}}\left(\left(\left(\square_{4 \mathrm{~T}} \xi_{1}\right) \supset_{4 \mathrm{~T}}\left(\square_{4 \mathrm{~T}}\left(\square_{4 \mathrm{~T}} \xi_{1}\right)\right)\right) \vee_{4 \mathrm{~T}}\left(\left(\square_{4 \mathrm{~T}} \xi_{1}\right) \supset_{4 \mathrm{~T}} \xi_{1}\right)\right) .
$$

Semantically, the modal box $\square_{4 \mathrm{~T}}$ is established by the class of Kripke structures with accessibility relation locally (that is, at each world) reflexive or transitive. This class includes the reflexive structures and the transitive structures, among others that are only locally reflexive or transitive. But it does not contain all the Kripke structures.

Clearly, there are more common features to $\square_{4}$ and $\square_{T}$ than those of the basic $\square_{\mathrm{K}}$. For instance, the latter does not fulfill the disjunction of the transitivity and the reflexivity axioms.

Within the setting of M4TL we may also investigate the properties of seemingly less interesting mixed constructors like $\left\lceil\neg_{4} \square_{\mathrm{T}}\right\rceil$. For instance,

$$
\vdash_{4 \mathrm{~T}}\left(\left(\left\lceil\neg_{4} \square_{\mathrm{T}}\right\rceil\left(\left\lceil\neg_{4} \square_{\mathrm{T}}\right\rceil \xi_{1}\right)\right) \supset_{4 \mathrm{~T}} \xi_{1}\right)
$$

but we refrain to delve into these issues. Indeed, for the purpose of combining modal logics one probably would drop such mixed constructors.

\section{Outlook}

While investigating different ways of combining logics and the reasons why they lead frequently to inconsistency, we came up with the idea of endowing each combined constructor with only the logical properties that are common to their components. To this end, we defined a new way of combining logics (meet-combination) where each constructor of the resulting language is a pair of constructors (of the same arity), one from each of the two original logics being combined. Each of the given logics is embedded in the resulting logic by pairing (meet-combining) it with the verum (of the same arity) from the other logic. In the resulting calculus one finds the rules corresponding to the original inference rules (via the embeddings mentioned above), rules imposing that the meetcombined constructors inherit the common properties of their components and only those common properties, and rules imposing the propagation of falsum. Each matrix of the resulting logic is just the product of a matrix from one of the original logics with a matrix of the other. In this way, the resulting logic is an enrichment of each of the two given logics (via the relevant embedding). 
The conservative and consistency preserving nature of the embeddings followed easily. We were also able to prove that soundness and (concrete) completeness are preserved by meet-combination.

Although we started with the axiomatization of the meet-combination (imposing on the combined constructors the common properties of their components) and only afterwards looked for an appropriate semantics, this turned out to be the product of the two given matrix semantics. Therefore, unexpectedly, we managed to obtain an axiomatization for the product of two matrix logics ${ }^{4}$.

For assessing what was achieved we looked with some detail into the meetcombination of classical and intuitionistic logics and the meet-combination of two modal logics, comparing in both cases the result with fibring. The fully conservative nature of meet-combination seems to be the key advantage of this new way of combining logics over other combination mechanisms previously reported in the literature, namely fibring.

It should be stressed that the conservativeness desideratum was achieved without loosing all the intuitions behind fibring. Each inherited connective (like $\left\lceil\square_{4} \mathrm{tt}_{\top}^{(1)}\right\rceil$ ) still behaves as expected since it inherits the laws imposed on $\square_{4}$ within the original logic whence it comes. The main difference between fibring and meet-combining logics concerns the non-inherited constructors (like $\left\lceil\square_{4} \square_{T}\right\rceil$ ). In fibring the sharing of $\square_{4}$ and $\square_{T}$ inherits the laws imposed on $\square_{4}$ and the laws imposed on $\square_{\mathrm{T}}$ (becoming the S4 box), while the meet-combination of these two modal boxes only inherits their common laws (becoming the newly discovered $\lceil 4 \mathrm{~T}\rceil$-box).

Meet-combination relies on the notion of meet-combined constructor, first proposed by us in [7] for studying the common properties of different connectives, say conjunction and disjunction. Otherwise, to our knowledge, not much work has been done on combining connectives (and other language constructors) outside the field of combined logics. A related idea should be mentioned nevertheless. In [2] a new connective is proposed which is defined only for the pairs of truth values where conjunction and disjunction agree.

The investigation of meet-combination should go on in several directions. First, a deeper study is necessary of interesting and relevant (from the point of view of applications) meet-combinations of logics. Second, so far we defined meet-combination only in the case of logics in the adopted universe (language of propositional nature, Hilbert calculus and matrix semantics). In order to widen the applicability of meet-combination, the work should be carried over to other kinds of semantics, such as non-deterministic matrices [1], possible-translations semantics [4], abstract valuations [3], and graph-theoretic interpretations [8], as well as to other kinds of deduction systems, such as sequent calculi. Furthermore, at some point the attempt should be made to leave the realm of propositional-based logics and address logics with variables and binding operators. Third, the investigation on transference properties of meet-combination should continue, namely concerning preservation of decidability. Fourth, the conservative nature of meet-combination may help in studying what happens when one wants to put together say a pure logic of negation with a pure logic

\footnotetext{
${ }^{4}$ As kindly pointed out by one of the reviewers.
} 
of disjunction without collateral effects.

\section{Acknowledgments}

The authors wish to express their gratitude to the reviewers for suggestions towards improving the readability of the paper. This work was partially supported by FCT and EU FEDER, namely via projects KLog PTDC/MAT/68723/2006, QSec PTDC/EIA/67661/2006 and AMDSC UTAustin/MAT/0057/2008, as well as under the GTF (Graph-Theoretic Fibring) and MCL (Meet-Combination of Logics) initiatives of SQIG at IT.

\section{References}

[1] A. Avron. Non-deterministic semantics for logics with a consistency operator. International Journal of Approximate Reasoning, 45(2):271-287, 2007.

[2] S. Blamey. Partial logic. In D. Gabbay and F. Guenthner, editors, Handbook of Philosophical Logic, 2nd Edition, volume 5, pages 261-353. Springer, 2002.

[3] C. Caleiro and R. Gonçalves. Abstract valuation semantics. Studia Logica, in print.

[4] W. A. Carnielli. Possible-translations semantics for paraconsistent logics. In D. Batens, C. Mortensen, G. Priest, and J. van Bendegem, editors, Frontiers of Paraconsistent Logic, Logic and Computation Series, pages 149-163. Research Studies Press, College Publications, 2000.

[5] D. M. Gabbay. Fibred semantics and the weaving of logics. I. Modal and intuitionistic logics. The Journal of Symbolic Logic, 61(4):1057-1120, 1996.

[6] A. Sernadas, C. Sernadas, and C. Caleiro. Fibring of logics as a categorial construction. Journal of Logic and Computation, 9(2):149-179, 1999.

[7] A. Sernadas, C. Sernadas, and J. Rasga. On combined connectives. Logica Universalis, in print.

[8] A. Sernadas, C. Sernadas, J. Rasga, and M. Coniglio. A graph-theoretic account of logics. Journal of Logic and Computation, 19:1281-1320, 2009.

[9] D. van Dalen. Intuitionistic logic. In D. Gabbay and F. Guenthner, editors, Handbook of Philosophical Logic, volume 5, pages 1-114. Springer, 2002. 2nd Edition. 